\title{
RESONANT EQUILIBRIUM CONFIGURATIONS IN QUASI-PERIODIC MEDIA: PERTURBATIVE EXPANSIONS
}

\author{
RAFAEL DE LA LLAVE, XIFENG SU, AND LEI ZHANG
}

\begin{abstract}
We consider 1-D quasi-periodic Frenkel-Kontorova models.
We study the existence of equilibria whose frequency (i.e. the inverse of the density of deposited material) is resonant with the frequencies of the substratum.

We study perturbation theory for small potential. We show that there are perturbative expansions to all orders for the quasi-periodic equilibria with resonant frequencies. Under very general conditions, we show that there are at least two such perturbative expansions for equilibria for small values of the parameter.

We also develop a dynamical interpretation of the equilibria in these quasi-periodic media. We show that equilibria are orbits of a dynamical system which has very unusual properties. We obtain results on the Lyapunov exponents of the dynamical systems, i.e. the phonon gap of the resonant quasi-periodic equilibria. We show that the equilibria can be pinned even if the gap is zero.
\end{abstract}

Quasi-periodic Frenkel-Kontorova models, resonant frequencies, equilibria, quasicrystals, Lindstedt series, counterterms

[2010] 70K43, 37J50, 37J40, 52C23

\section{INTRODUCTION}

The goal of this paper is to formulate the theory of resonant equilibria in quasi-periodic Frenkel-Kontorova models. We argue that these equilibria play an important role in the phenomenon of pinning (the equilibria that survive after an external force is applied). In periodic Frenkel-Kontorova models, the role of resonances in pinning is well established.

We recall that in Frenkel-Kontorova models[BK04, Sel92], one considers configurations given by a sequence of real numbers (think of the position of a sequence of particles deposited on a 1-D material). The (formal) energy of the system is the sum of a term of interaction between nearest neighbors of the deposited material and a term modeling interaction with the media. In

L. Z and R. L. supported by NSF grant DMS-1500943.

X. S supported by National Natural Science Foundation of China (Grant No. 11301513) and "the Fundamental Research Funds for the Central Universities". 
the quasi-periodic Frenkel-Kontorova models studied here, the interacting potential will be a quasi-periodic function of the position reflecting that the medium is quasi-periodic. We will be interested in equilibria, i.e., configurations such that the derivatives of the (formal) energy with respect to the position of each of the particles vanish. We note that even if the energy is a formal sum, the equilibrium equations are well defined. More details of the models will be discussed in Section 2 ,

In [SdlL12b, SdlL12a], one can find a rigorous mathematical theory of quasi-periodic solutions whose frequency is not resonant (indeed Diophantine) with the frequencies of the substratum. The rigorous theory of [SdlL12b, SdlL12a] also leads to efficient algorithms that can compute these quasiperiodic solutions arbitrarily close to their breakdown. Implementations of these algorithms and investigation of the phenomena at breakdown appear in [BdlL13]. The paper [SdlL12b], studies models with nearest neighbor interaction while [SdlL12a] studies the case of long range interactions.

Our motivation is to study the phenomena of "depinning". When we add an external force (no matter how small) to the model, many quasi-periodic solutions disappear. Nevertheless, there are still other quasi-periodic solutions survive. This physically corresponds to the deposited material rearranges itself to withstand the force. It is well known in the periodic FrenkelKontorova models that the solutions which persist under forcing are resonant with the media. The papers [SdlL12b, SdlL12a] also show that, in the quasi-periodic case, the smooth non-resonant solutions do not exist when there is an external force. Hence we are interested in resonant solutions in the quasi-periodic models.

In this paper, we show that there exist at least two formal power series in the amplitude of the coupling describing resonant solutions even in the presence of external forces. The delicate analytic question of convergence of these series will be pursued in [dlLSZ15]. We also develop a dynamical interpretation of the equilibria as orbits of a dynamical system. Using this dynamical interpretation, we show that the phonon gap of quasi-periodic equilibria is zero.

We hope that this paper can lay the ground work for future explorations. We will use rigorous mathematical tools to study the convergence of the formal series developed here in [dlLSZ15]. We will also use numerical methods to explore in a non-rigorous but more quantative way some of the phenomena discussed here.

Equilibria in quasi-periodic media with a resonant frequency have been investigated numerically in [vEFRJ99, vEFJ01, vEF02]. These papers also studied the phonon gap and found it to vanish when there are smooth solutions (in agreement with the results here). 
The variational and topological methods that have been proved useful in the periodic case do not extend to the quasi-periodic case in its full strength. Several interesting counterexamples are in [LS03, Fed75]. Partial results are in [GGP06, AP10, GPT13, KO13].

\section{Models CONSIDERED AND FORMULATION OF THE PROBLEM}

We consider models of deposition in a quasi-periodic one-dimensional medium.

If $x_{n} \in \mathbb{R}$ denotes the position of the $n$-th particle of the deposited material, the state of the system is specified by a configuration, i.e. a sequence $\left\{x_{n}\right\}_{n \in \mathbb{Z}}$. We associate the following formal energy to a configuration of the system

$$
\mathscr{S}(x)=\frac{1}{2} \sum_{n \in \mathbb{Z}}\left(x_{n+1}-x_{n}-a\right)^{2}-V\left(x_{n} \alpha\right)-\lambda x_{n}
$$

where $V: \mathbb{T}^{d} \rightarrow \mathbb{R}$ is an analytic function, $\alpha \in \mathbb{R}^{d}$ is an irrational vector and $a, \lambda$ are some real numbers.

A natural example often used is

$$
\mathscr{S}(x)=\frac{1}{2} \sum_{n \in \mathbb{Z}}\left(x_{n+1}-x_{n}-a\right)^{2}+A \cos x_{n}+B \cos \left(\sqrt{2} x_{n}+\phi\right)-\lambda x_{n}
$$

which corresponds to $\alpha=(1, \sqrt{2})$ and $V\left(\theta_{1}, \theta_{2}\right)=A \cos \theta_{1}+B \cos \left(\theta_{2}+\phi\right)$.

The term $\left(x_{n+1}-x_{n}-a\right)^{2}$ represents the interaction among neighboring deposited atoms. The term $V\left(x_{n} \alpha\right)$ represents the interaction with the substratum. The interaction at position $x \in \mathbb{R}$ is the quasi-periodic function $V(x \alpha)$. This models that the substratum is quasi-periodic. The term $\lambda x_{n}$ has the interpretation of a constant field applied to the model. In the case of deposited materials, we can imagine that the sample is tilted and $\lambda$ is the component of the gravity. The physical meaning of the equilibria corresponding to $\lambda \neq 0$ is that, when we apply a small external field, the configurations rearrange themselves so that they can respond to the force and do not slide. Of course, when the force is strong enough, no rearrangement is possible and all the configurations slide. This is the microscopic origin of static friction.

In the case of periodic media, the pinned solutions are known to correspond to resonant frequencies. Since the resonant tori do not survive, it is reasonable to consider resonant frequencies.

The existence of external forces $\lambda$ is a very important novelty with respect to the previous papers [SdlL12b, SdlL12a]. It was shown in [SdlL12b, SdlL12a] that if there is non-resonant quasi-periodic solution, then $\lambda=0$. In our case, we will show how to construct quasi-periodic equilibria with 
nontrivial $\lambda$ and will show how to compute perturbatively the range of such $\lambda$ for which solutions with a prescribed resonant frequency exist.

Without any loss of generality, we can assume that

$$
k \cdot \alpha \notin \mathbb{N} \quad \forall k \in \mathbb{Z}^{d}-\{0\} .
$$

If there existed a resonance $k \cdot \alpha=0$, we could just use less frequencies to express the quasi-periodic function.

2.1. Equilibrium equations. A configuration is in equilibrium if the forces acting on all the particles vanish. Equivalently, the derivatives of the energy with respect to the position of the particles vanish. That is,

$$
\frac{\partial \mathscr{S}}{\partial x_{n}}(x)=0 \quad \forall n \in \mathbb{Z} .
$$

In the model (1), the equilibrium equations are

$$
x_{n+1}+x_{n-1}-2 x_{n}+\partial_{\alpha} V\left(x_{n} \alpha\right)+\lambda=0 \quad \forall n \in \mathbb{Z}
$$

where $\partial_{\alpha}=\alpha \cdot \nabla$ and $\nabla$ is the usual gradient.

Note that even if the energy (1) is just a formal sum, the equilibrium equations (4) are well defined equations.

It is very tempting to consider (4) as a dynamical system, so that we obtain $x_{n+1}$ as a function of $x_{n}$ and $x_{n-1}$. This system has very unusual properties. This will be pursued in Section 5 .

2.2. Quasi-periodic configurations, hull functions. In this paper, we will be interested in quasi-periodic solutions of frequency $\omega \in \mathbb{R}$.

These are configurations of the form

$$
x_{n}=n \omega+h(n \omega \alpha) \text {, }
$$

where $h: \mathbb{T}^{d} \rightarrow \mathbb{R}$.

A configuration given by a hull function (5) satisfies the equilibrium equation (4) if and only if the hull function $h$ satisfies

(6) $h(n \omega \alpha+\omega \alpha)+h(n \omega \alpha-\omega \alpha)-2 h(n \omega \alpha)+\partial_{\alpha} V(n \omega \alpha+\alpha h(n \omega \alpha))+\lambda=0$.

The equation (6) was considered in [SdlL12b, SdlL12a] when $\omega \alpha$ is Diophantine (in particular, $n \omega \alpha$ is dense in the torus $\mathbb{T}^{d}$ ).

In our case, $n \omega \alpha$ will not be dense on the $d$-dimensional torus (see Section 2.3) and the equilibrium equations we will derive are different from those in [SdlL12b, SdlL12a]. 
2.3. Resonances. The goal of this paper is to study situations when there are $k \in \mathbb{Z}^{d}-\{0\}$ and $m \in \mathbb{Z}$ such that

$$
k \cdot \omega \alpha-m=0 .
$$

When (7) holds we say that $(k, m)$ is a discrete resonance for $\omega \alpha$ and we refer to the pair $(k, m)$ as a resonance.

Remark 1. Note that these discrete resonances (7) are different from the resonances of the media we excluded before $\left(k \cdot \alpha \neq 0, \forall k \in \mathbb{Z}^{d}-\{0\}\right)$.

Remark 2. If

$$
k \cdot \alpha \neq 0 \quad \forall k \in \mathbb{Z}^{d} \backslash\{0\},
$$

given any $k_{0} \in \mathbb{Z}^{d} \backslash\{0\}, m \in \mathbb{Z}$ we have that $\omega=-m /\left(k_{0} \cdot \alpha\right)$ is a resonant frequency. Since $k_{0} \cdot \alpha$ can be arbitrarily large, we see that the set of resonant frequencies is dense on the real line. Of course, once we fix $\alpha$, the set of resonant $\omega$ is a countable set.

2.3.1. Multiplicity of a resonance. Clearly, if $(k, m),(\tilde{k}, \tilde{m})$ are discrete resonances so is $(k+\tilde{k}, m+\tilde{m})$.

In mathematical language,

$$
\mathscr{M}_{\omega \alpha}=\left\{(k, m) \in \mathbb{Z}^{d} \times \mathbb{Z}: k \cdot \omega \alpha-m=0\right\}
$$

is a $\mathbb{Z}$-module called the resonance module for $\omega$.

We denote by $l(\omega)=\operatorname{dim}\left(\mathscr{M}_{\omega \alpha}\right)$ the dimension of the resonance module and we call it the multiplicity of the resonance. The meaning of $l(\omega)$ is the number of independent resonances. We can find $\left(k_{1}, m_{1}\right), \ldots,\left(k_{l}, m_{l}\right)$ in such a way that all resonances can be expressed as combinations of the basic resonances (and also no other set of basic resonances with smaller number of elements will allow to express all the resonances).

2.3.2. Only resonances of multiplicity 1 appear in the models (1). In Hamiltonian mechanics for systems with $d$ degrees of freedom, one can find resonances of all multiplicities up to $d$. As we will see later, in Section 5, one can give a dynamical interpretation of the equilibrium equations as a dynamical system in $d+1$ dimensions. Nevertheless, in our models only $l=1$ appears independently of the number of degrees of freedom. This highlights that the problem here is different from the Hamiltonian problem.

Proposition 1. If $\omega \alpha$ is resonant, i.e. $\mathscr{M}_{\omega \alpha} \neq\{0\}$, then $l(\omega)=1$.

Proof. Note that

$$
k_{1} \cdot \omega \alpha-m_{1}=k_{2} \cdot \omega \alpha-m_{2}=0
$$

implies (because $m_{1} \neq 0, m_{2} \neq 0$ because of (3))

$$
\omega=\frac{m_{1}}{k_{1} \cdot \alpha}=\frac{m_{2}}{k_{2} \cdot \alpha}
$$


and therefore

$$
\alpha \cdot\left(k_{1} m_{2}-k_{2} m_{1}\right)=0
$$

and, because $\alpha$ is non-resonant (3) we have

$$
k_{1} m_{2}=k_{2} m_{1}
$$

Therefore, the two resonant vectors are related.

2.3.3. The intrinsic frequencies. When $\omega \alpha$ is resonant, we can find a matrix $B \in S L(d, \mathbb{Z}), \Omega \in \mathbb{R}^{d-1}, L \in \mathbb{Z}^{d}$ in such a way that

$$
B \omega \alpha=(\Omega, 0)+L \quad \text { with } \Omega \cdot k \notin \mathbb{Z} \text { for } k \in \mathbb{Z}^{d-1}-\{0\} .
$$

We will refer to $\Omega$ 's as the intrinsic frequencies. They are essentially unique, i.e., unique up to changes of basis in $\mathbb{R}^{d-1}$ given by a matrix in $S L(d-1, \mathbb{Z})$.

In this case, the set $\{n \omega \alpha\}_{n \in \mathbb{Z}}$ has a closure which is a $d-1$ dimensional torus. This torus is invariant under the translation $T_{\omega \alpha}$. If we stay in this $d-1$ dimensional torus, $T_{\omega \alpha}$ can be described as $T_{\Omega}$. The torus $\mathbb{T}^{d}$ is foliated by these $\mathbb{T}^{d-1}$ indexed by another parameter $\eta \in \mathbb{T}^{1}$. We will write a point in $\mathbb{T}^{d}$ as $(\psi, \eta)$ where $\psi$ is the coordinate corresponding to the position in $\mathbb{T}^{d-1}$. The coordinate $\eta$ selects the $d-1$ torus we are considering.

2.4. Quasi-periodic equilibria with resonant frequencies. The natural notion of the hull functions in the resonant case would be to assume that the equilibrium solutions have the form

$$
x_{n}=n \omega+v(n \Omega)
$$

with $v: \mathbb{T}^{d-1} \rightarrow \mathbb{R}$.

Note that the physical meaning of $\omega$ is still the mean spacing of the solutions (i.e., an inverse density). The term $v(n \Omega)$ represents fluctuations that can be parameterized in terms of the intrinsic frequency $\Omega$. Of course, we could represent them in terms of the original frequencies, but it is more natural to change variables so that they become a part of the equation.

We will refer to the $\eta$ variable as the transversal phase. The resonant solutions considered here, cover densely a torus of codimension one. The one-dimensional variable $\eta$ measures the position of these codimension-one tori on the configuration space $\mathbb{T}^{d}$ corresponding to the internal phases of $V$.

If we substitute the parameterization (9) into the equilibrium equation, we obtain that the equilibrium equation (4) is equivalent to:

$$
v(n \Omega+\Omega)+v(n \Omega-\Omega)-2 v(n \Omega)+\partial_{\alpha} V(n \omega \alpha+\alpha v(n \Omega))+\lambda=0 .
$$


If we furthermore introduce the notation $\partial_{\alpha} V(\theta)=W(B \theta)$ and $B \alpha=$ $\beta$, and observe that the $n \Omega$ is dense on $\mathbb{T}^{d-1}$, we see that for continuous functions $v,(10)$ is equivalent to:

$$
v(\psi+\Omega)+v(\psi-\Omega)-2 v(\psi)+W((\psi, 0)+\beta v(\psi))+\lambda=0 .
$$

Note that we can also consider solutions of the form

$$
x_{n}=n \omega+v\left(n \Omega+\xi_{1}\right)+\xi_{2}
$$

for any fixed $\xi_{1}$ and $\xi_{2}$. This will give us freedom to add the transversal phase $\eta$ as an additional parameter in (11). So the equilibrium equation we will consider is

$$
v(\psi+\Omega)+v(\psi-\Omega)-2 v(\psi)+W((\psi, \eta)+\beta v(\psi))+\lambda=0 .
$$

By simple calculations, it can be shown that (13) is equivalent to (4) when the hull function is of the form (12) and $B \alpha \xi_{2}=\left(\xi_{1}, \eta\right)$.

Remark 3. Because $\beta$ has components both in the $\psi$ and the $\eta$ directions, the equation (13) cannot be considered as a parameterized version of the equations considered in [S[S1L12b]. As we will see, the symmetries of the equation involve transformations that mix the dependence in $\psi$ and in $\eta$.

2.5. The symmetries of the invariance equation (13). The equation (13) possesses remarkable symmetries that make the solutions not unique. These symmetries lead to Ward identities. In contrast with the case of non-resonant solutions, the group of symmetries is infinite dimensional. In [dlL08, SdlL12b, SdlL12a] these symmetries are used to develop a KAM method.

The main observation is that if $(v, \lambda)$ is a solution of (13), then, for every $\iota(\eta): \mathbb{T}^{1} \rightarrow \mathbb{R}$, the pair $(\tilde{v}, \tilde{\lambda})$ is also a solution of (13) where we denote $\beta=\left(\beta_{\psi}, \beta_{\eta}\right)$ and $\tilde{v}, \tilde{\lambda}$ are defined by:

$$
\begin{aligned}
\tilde{v}(\psi, \eta) & =v((\psi, \eta)+\iota(\eta) \beta)+\iota(\eta), \\
\tilde{\lambda}(\eta) & =\lambda\left(\eta+\iota(\eta) \beta_{\eta}\right) .
\end{aligned}
$$

Notice that the symmetry (14) involves changing not only the argument $\psi$ but also the argument $\eta$. Note the space of symmetries of the equation is not just a finite dimensional space but rather an infinite dimensional space of functions.

2.6. A normalization of the solutions of the invariance equation (13). For later applications, it will be useful to have local uniqueness of the solutions (e.g. to discuss smooth dependence on parameters, perturbative expansions on parameters). Hence we impose the normalization

$$
\int_{\mathbb{T}^{d-1}} v(\psi, \eta) d \psi=0
$$


Since the symmetry (14) involves changes of arguments, giving a $v_{\eta}$, finding the $\iota(\eta)$ that accomplishes the normalization involves solving the implicit equation

$$
I\left(\eta+\beta_{\eta} \iota(\eta)\right)+\iota(\eta)=0
$$

where $I(\eta) \equiv \int_{\mathbb{T}^{d-1}} v(\psi, \eta) d \psi$.

If $I$ and its derivative are small, one can solve eqrefimplicit using implicit function theorem.

2.7. Diophantine condition. In contrast with KAM theory, we will not need very delicate estimates on the solutions and hence, we can deal with very general Diophantine conditions. We will assume that $\Omega$ satisfies

$$
\lim _{N \rightarrow \infty} \frac{1}{N} \sup _{|k| \leq N, m \in \mathbb{Z}}|\ln | k \cdot \Omega-m||=0 .
$$

Note that the condition (17) is much weaker than the usual Diophantine conditions and even than the Bjruno-Rüssmann conditions. The condition(17) is the natural condition in the study of existence of series to all orders.

The following proposition shows that the sets of frequencies we are considering are abundant.

Fix a vector $k \in \mathbb{Z}^{d} \backslash\{0\}, m \in \mathbb{Z} \backslash\{0\}$ and assume without loss of generality that there is no common divisor in the components of $k$. For any $\alpha \in \mathbb{R}^{d}$ satisfying $\alpha \cdot k \neq 0$, we can find a unique $\omega$ such that $\alpha \cdot k \omega-m=0$. Fix a $B_{k} \in S L(d, \mathbb{Z})$ such that the last row of $B$ equals $k$ (It's possible to find such $B$ since the components of $k$ has no common divisor). Then, let $B_{k} \alpha \omega=(\Omega, 0)+m$. Hence, for any $k, m$ and $\alpha$ satisfying $\alpha \cdot k \neq 0$, we can define $\Omega$ as a function of $\alpha$. Denote $\Omega=F_{k, m}(\alpha)$.

Proposition 2. The set of $\alpha$ for which $F_{k, m}(\alpha)$ satisfies (17) for all $k, m$ is of full measure in $\mathbb{R}^{d}$. In other words, for a full measure set of medium frequencies, we can find a countable many resonant frequencies that lead to intrinsic frequencies satisfying (17).

Proof. Since countable intersections of sets of full measure are of full measure, to prove Proposition 2 it suffices to show that for a fixed $k, m$ as above, the set $\left\{\alpha \in \mathbb{R}^{d} \mid \alpha \cdot k \neq 0\right.$ and $F_{k, m}(\alpha)$ satisfies (17) $\}$ is of full measure.

Because the set of $\Omega$ 's which satisfy (17) is of full measure on $\mathbb{R}^{d-1}$ and the linear map $B_{k}$ is differentiable and surjective, the preimage of the set of $\Omega$ 's that satisfy (17) under $B_{k}$ is also of full measure in the hyperplane $\Gamma=\{\gamma \mid \gamma \cdot k-m=0\}$. Denote the set of preimages as $\Gamma^{\prime}$. Then any nonzero scaling of an element of $\Gamma^{\prime}$ will give an $\alpha$ we want, which also form a full measure set in $\mathbb{R}^{d}$. 


\section{FUNCTION SPACES AND LINEAR ESTIMATES}

The main tool that we will use to construct perturbation theories is the solution of cohomology equations.

We denote

$$
D_{\rho}=\left\{\theta \in \mathbb{C}^{d} / \mathbb{Z}^{d}|| \operatorname{Im}\left(\theta_{i}\right) \mid<\rho\right\}
$$

and denote the Fourier expansion of a periodic mapping $v(\psi, \eta)$ on $D_{\rho}$ by

$$
v(\psi, \eta)=\sum_{k \in \mathbb{Z}^{d}} v_{k} e^{2 \pi i k \cdot(\psi, \eta)}
$$

where $\cdot$ is the Euclidean scalar product in $\mathbb{C}^{d}$ and $v_{k}$ are the Fourier coefficients.

We denote by $\mathscr{A}_{\rho}$ the Banach space of analytic functions on $D_{\rho}$ which are real for real argument and extend continuously to $\overline{D_{\rho}}$. We make $\mathscr{A}_{\rho}$ a Banach space by endowing it with the supremum norm:

$$
\|v\|_{\rho}=\sup _{(\psi, \eta) \in \overline{D_{\rho}}}|v(\psi, \eta)| .
$$

These Banach spaces of analytic functions are the same spaces as in [Mos67].

We will consider equations of the form

$$
v(\psi+\Omega, \eta)-v(\psi, \eta)=\phi(\psi, \eta)
$$

where $\psi \in \mathbb{T}^{d-1}$.

To simplify our notations, we will denote $v(\psi+\Omega)$ and $v(\psi-\Omega)$ as $v_{+}$ and $v_{-}$, respectively. Similar notations will be used for other functions. We also use $T$ to represent the translation operators, i.e., $T_{\Omega} v(\psi)=v(\psi+\Omega)$.

Lemma 1. Let $\phi \in \mathscr{A}_{\rho}\left(\mathbb{T}^{d}\right)$ be such that

$$
\int_{\mathbb{T}^{d-1}} \phi(\psi, \eta) d \psi=0
$$

for all $\eta$.

Assume that $\Omega$ satisfies the assumption (17).

Then, for a fixed $\eta$, there exists a unique solution $v_{\eta}$ of (18) which satisfies

$$
\int_{\mathbb{T}^{d-1}} v(\psi, \eta) d \psi=0
$$

The solution $v \in \mathscr{A}_{\rho^{\prime}}$ for any $\rho^{\prime}<\rho$ and we have

$$
\|v\|_{\rho^{\prime}} \leq C\left(\rho, \rho^{\prime}\right)\left\|\phi_{\eta}\right\|_{\rho} .
$$

Furthermore, any distribution solution of (18) differs from the solution claimed before by a constant.

If $\phi$ is such that it takes real values for real arguments, so does $v$. 
If we consider now the dependence in $\eta$, we have that $v \in \mathscr{A}_{\rho^{\prime}}\left(\mathbb{T}^{d}\right)$ and

$$
\|v\|_{\rho^{\prime}} \leq C\left(\rho, \rho^{\prime}\right)\|\phi\|_{\rho}
$$

Proof. We note that, as it is well known that obtaining $v$ solving (18) for given $\phi$ is very explicit in terms of Fourier coefficients. If

$$
\phi(\psi, \eta)=\sum_{k \neq 0} \hat{\phi}_{k}(\eta) e^{2 \pi i k \cdot \psi}=\sum_{k \neq 0, m} \hat{\phi}_{k, m} e^{2 \pi i(k \cdot \psi+m \eta)}
$$

then, $v$ is given by

$$
v(\psi, \eta)=\sum_{k \neq 0} \hat{\phi}_{k}(\eta)\left(e^{2 \pi i k \cdot \Omega}-1\right)^{-1} e^{2 \pi i k \cdot \psi}=\sum_{k \neq 0, m} \hat{\phi}_{k, m}\left(e^{2 \pi i k \cdot \Omega}-1\right)^{-1} e^{2 \pi i(k \cdot \psi+m \eta)} .
$$

Using Cauchy estimates for the Fourier coefficients $\left|\hat{\phi}_{k, m}\right| \leq \exp (-2 \pi \rho(|k|+$ $|m|))\|\phi\|_{\rho}$ and that $\left|e^{2 \pi k \cdot \Omega}-1\right|^{-1} \leq C \operatorname{dist}(k \cdot \Omega, \mathbb{Z})^{-1}$ and the assumption (17), we obtain that

$$
\begin{aligned}
\|v\|_{\rho^{\prime}} & \leq C \sum_{k \neq 0, m} \exp (-2 \pi \rho(|k|+|m|))\|\phi\|_{\rho} \operatorname{dist}(k \cdot \Omega, \mathbb{Z})^{-1}\left\|e^{2 \pi i(k \cdot \psi+m \eta)}\right\|_{\rho^{\prime}} \\
& \leq C\|\phi\|_{\rho} \sum_{k \neq 0, m} \exp (-2 \pi \rho(|k|+|m|)) \operatorname{dist}(k \cdot \Omega, \mathbb{Z})^{-1} \exp \left(2 \pi \rho^{\prime}(|k|+|m|)\right) \\
& \leq C\left(\rho, \rho^{\prime}\right)\|\phi\|_{\rho} .
\end{aligned}
$$

\section{LINDSTEDT SERIES FOR QUASI-PERIODIC SOLUTIONS WITH RESONANT FREQUENCIES}

The goal of this section is to study (13) perturbatively when the nonlinear term is small. Hence, we will write (13) with a small parameter $\varepsilon$

$$
v(\psi+\Omega, \eta)+v(\psi-\Omega, \eta)-2 v(\psi, \eta)+\epsilon W((\psi, \eta)+\beta v(\psi, \eta))+\lambda(\eta)=0 .
$$

We will find $v(\psi, \eta), \lambda(\eta)$ solving (21) and (15) in the sense of formal power series in $\epsilon$. In this paper, we will not consider the problem of whether these series converge or represent a function. This will be studied in more details in [dlLSZ15].

Since one may want to find solutions correspond to $\lambda=0$ (or $\lambda=\lambda^{*}$ with $\left|\lambda^{*}\right|$ small $)$, it is important for us to keep track of $\frac{\partial \lambda}{\partial \eta}(\eta, \epsilon)$ in order to solve $\lambda(\eta, \epsilon)=0$ by implicit function theorem. 
Following the standard perturbative procedure we will write

$$
\begin{aligned}
& v=\sum_{n=0}^{\infty} \epsilon^{n} v^{n}, \\
& \lambda=\sum_{n=0}^{\infty} \epsilon^{n} \lambda^{n} .
\end{aligned}
$$

Here $v^{n}$ and $\lambda^{n}$ are coefficients of $\epsilon^{n}$, not powers of $v$ or $\lambda$. Substitute (22) in (21) and equate powers of $\epsilon$.

Of course, carrying out this procedure for $n \leq N$ will require that $\Omega$ satisfies some Diophantine properties as well as some differentiability assumptions.

Equating the coefficients of $\epsilon^{0}$ in (21) we obtain

$$
v^{0}(\psi+\Omega, \eta)+v^{0}(\psi-\Omega, \eta)-2 v^{0}(\psi, \eta)+\lambda^{0}(\eta)=0 .
$$

Hence, if $\Omega$ satisfies the condition (17) we see that $v^{0}$ is constant, $\lambda^{0}=0$ and imposing the normalization (15) we obtain $v^{0}=0$.

Matching coefficients of $\epsilon^{1}$ in both sides of (21) we obtain

$$
v^{1}(\psi+\Omega, \eta)+v^{1}(\psi-\Omega, \eta)-2 v^{1}(\psi, \eta)+W(\psi, \eta)+\lambda^{1}(\eta)=0 .
$$

We see that, using the theory in Section 3, to have analytic $v^{1}$ solving (24), it is necessary and sufficient to have

$$
\lambda^{1}(\eta)=-\int_{\mathbb{T}^{d-1}} W(\psi, \eta) d \psi
$$

Then, $v^{1}, \lambda^{1}$ can be determined uniquely up to a constant from (24). In fact, in Fourier series, the equation for $v^{1}, \lambda^{1}$ is

$$
v_{k}^{1} 2(\cos (2 \pi k \Omega)-1)=-W_{k}-\delta_{0, k} \lambda^{1},
$$

where $\delta_{0, k}$ is the Kronecker delta. In particular, the constant in $v^{1}$ is determined by the normalization (15).

Proceeding to higher order follows the same pattern. We see that matching the terms of order $\epsilon^{n}$ in (21) we obtain

$$
v^{n}(\psi+\Omega, \eta)+v^{n}(\psi-\Omega, \eta)-2 v^{n}(\psi, \eta)+R^{n}(\psi, \eta)+\lambda^{n}(\eta)=0,
$$

where $R^{n}$ is a polynomial expression in $v^{1}, \ldots, v^{n-1}$ with coefficients which are derivatives with respect to $\psi$ of $W((\psi, \eta)+\beta v(\psi, \eta))$. This polynomial can be computed explicitly because it is given by

$$
R^{n}=\left.\frac{1}{(n-1) !} \frac{d^{n-1}}{d \epsilon^{n-1}} W\left((\psi, \eta)+\beta \sum_{j=0}^{n-1} v^{j}(\psi, \eta)\right)\right|_{\epsilon=0}
$$


and these are well known formulae. We also note that, from the algorithmic point of view there are efficient ways to compute $R^{N}$ using methods of "automatic differentiation" [Har11, $\left.\mathrm{BCH}^{+} 06\right]$.

Since $R^{n}$ can be computed explicitly, (27) can be solved the same way as (24).

We have therefore established

Theorem 1. Assume that $\Omega$ satisfies (17) and that $W: D_{\rho} \rightarrow \mathbb{C}$ is an analytic function. We can find formal power series solutions in $\epsilon$ of the form (22) solving the equation (21). For any $0<\delta<\rho$, each of the terms $v^{n}(\psi, \eta)$ is analytic in $D_{\rho-\delta}$. If $W$ takes real values for real values, then so do $v^{n}$, and $\lambda$ is real.

4.1. The auxiliary equation. Now, we turn to the problem of studying the equation

$$
\lambda(\eta, \epsilon)=\lambda^{*}
$$

We expect to obtain a solution $\eta^{*}(\epsilon)$ provided that (29) satisfies some nondegeneracy conditions.

Having solution of (29) to order 1 in $\epsilon$, amounts to

$$
\lambda^{1}(\eta)=0 .
$$

That is, we need to find $\eta$ such that

$$
\int_{\mathbb{T}^{d-1}} W((\psi, \eta)) d \psi=0
$$

Theorem 2. The equation (30) has always two solutions.

Proof. Since

$$
\int_{\mathbb{T}^{d-1}} W((\psi, \eta)) d \psi=\int_{\mathbb{T}^{d-1}}\left(\partial_{\alpha} V\right)\left(B^{-1}(\psi, \eta)\right) d \psi,
$$

if we integrate again with respect to $\eta$ we obtain

$$
\int_{\mathbb{T}} \int_{\mathbb{T}^{d-1}} W\left((\psi, \eta) d \psi d \eta=\int_{\mathbb{T}^{d}}\left(\partial_{\alpha} V\right)\left(B^{-1}(\psi, \eta)\right) d \psi d \eta=0 .\right.
$$

Hence the function of $\eta$ given by $\int_{\mathbb{T}^{d-1}} W((\psi, \eta) d \psi$ is a continuous periodic function of $\eta$ with zero average. Therefore, it has at least two zeros. We also note that there are open sets of perturbations where there are $4,6, \cdots$ zeros.

Denote one of these solutions of (30) as $\eta^{*}$.

A sufficient condition that ensures that we can solve the equation (29) to all orders is that

$$
\left.\frac{\partial}{\partial \eta} \lambda^{1}(\eta, \epsilon)\right|_{\eta=\eta^{*}, \epsilon=0} \neq 0
$$


More explicitly,

$$
\int_{\mathbb{T}^{d}} \frac{\partial}{\partial \eta}\left(\partial_{\alpha} V\right)\left(B^{-1}(\psi, \eta)\right) d \psi d \eta \neq 0 .
$$

Then, the implicit function theorem for power series [Car95, Die71] gives us that we can indeed find $\eta^{*}(\epsilon)$.

Similarly, we can solve the equation $\lambda(\eta)=\lambda^{*}$ provided that $\left|\lambda^{*}\right|$ is sufficiently small.

Therefore, we have established

Theorem 3. Assume that $\Omega \in \mathscr{D}(v, \tau)$ as defined in (17), that $W$ is an analytic function, and that (33) holds, we can find formal power series $\eta_{\epsilon}$ in $\epsilon$ so that $v_{\eta_{\epsilon}}$ is the solution of (21).

Clearly, since the function $\lambda^{n}(\eta)$ are bounded, if $\lambda^{*}$ - the physical force - is large enough, there is no solution. This has a clear physical meaning. If we increase the external force but keep it small, the system can react by changing the transversal phase. If the force increases beyond a threshold, the system cannot react by adapting the phase. Hence, the equilibrium breaks down. In this paper, we are not considering the dynamics of the model, only the equilibria (our models for the energy include only the potential energy of the configuration and not any kinetic energy). One can, however, expect that, if there was some dynamics, the equilibria considered here could slide.

Of course, the sufficient condition (33) is far from being necessary and there are many other conditions that are enough.

Proposition 3. Assume that $\Omega$ satisfies (17), that $W((\psi, \eta)+\beta v(\psi, \eta))$ is an analytic function, and that (33) holds.

Assume that $\eta^{*}$ is such that for some $m \in \mathbb{N}$ we have

$$
\begin{aligned}
& \lambda^{i}\left(\eta^{*}\right)=0, \quad i=1, \ldots 2 m \\
& \lambda^{2 m+1}\left(\eta^{*}\right) \neq 0 .
\end{aligned}
$$

Then, we can find formal power series $\eta_{\epsilon}$ in $\epsilon$ so that $v\left(\psi, \eta_{\epsilon}\right)$ is the solution of (21).

The proof is again an application of the implicit function theorem for power series.

\section{A DYNAMICAL INTERPRETATION OF THE EQUILIBRIUM EQUATIONS OF FRENKEL-KONTOROVA MODELS}

In this section, we present a dynamical interpretation of the equilibrium equations (4) in Frenkel-Kontorova models. 
Even if the dynamical interpretation is possible for finite range interactions, we see that adding another small interaction of longer range is a singular perturbation (even the dimension of the phase space changes). Whereas, for the methods in this paper, adding a small term in the longer range is a regular perturbation of the same order.

A straightforward way of transforming the equilibrium equation

$$
x_{n+1}+x_{n-1}-2 x_{n}+\epsilon \partial_{\alpha} V\left(x_{n} \alpha\right)+\lambda=0 \quad \forall n \in \mathbb{Z}
$$

into a dynamical system is setting

$$
\begin{aligned}
y_{n} & =\left(x_{n}, x_{n-1}\right) \\
y_{n+1} & =\left(2 y_{n}^{1}-y_{n}^{2}-\epsilon \partial_{\alpha} V\left(\alpha y_{n}^{1}\right)-\lambda, y_{n}^{1}\right) .
\end{aligned}
$$

However, (36) is not very useful because we have to consider it as a map of $\mathbb{R}^{2}$ and the term $\partial_{\alpha} V\left(\alpha y_{n}^{1}\right)$ does not make apparent that it is periodic in $\alpha y_{n}^{1}$.

A more natural formulation is obtained by observing that the equation (4) is equivalent to the system on $\mathbb{T}^{d} \times \mathbb{R}$

$$
\begin{aligned}
& p_{n+1}=p_{n}-\epsilon \partial_{\alpha} V\left(q_{n}\right)-\lambda \\
& q_{n+1}=q_{n}+\alpha p_{n+1},
\end{aligned}
$$

where $q_{n} \in \mathbb{T}^{d}, p_{n} \in \mathbb{R}$. (Just multiply (4) by $\alpha$ and use the substitution $p_{n}=x_{n}-x_{n-1}, q_{n}=\alpha x_{n}$. Note that (4) is equivalent to

$$
\left(x_{n+1}-x_{n}\right)-\left(x_{n}-x_{n-1}\right)+\epsilon \partial_{\alpha} V\left(\alpha x_{n}\right)+\lambda=0
$$

hence, we obtain the first equation.)

We will write the mapping (37) as

$$
\left(p_{n+1}, q_{n+1}\right)=F_{\varepsilon, \lambda}\left(p_{n}, q_{n}\right) .
$$

Note that (37) is typographically very similar to the standard map [Chi79] or to analogues introduced for volume preserving maps. Nevertheless, there are significant differences (besides the different dimensions).

A very crucial difference between (38) and the generic volume preserving maps is that $q_{n+1}-q_{n}$ is always a multiple of $\alpha$ (see (37)). So that the two dimensional leaves

$$
\mathcal{M}_{q_{0}}=\left\{\left(p, q_{o}+\alpha t\right) \mid p, t \in \mathbb{R}\right\}
$$

are preserved. Note that each of the leaves $\mathcal{M}_{q_{0}}$ is dense in the $d+1$ dimensional phase space.

The mapping (37) clearly preserves the volume form $d p \wedge d q_{1} \wedge \ldots \wedge q_{d}$ since it is the composition of

$$
\begin{aligned}
& p_{n+1}=p_{n}-\epsilon \partial_{\alpha} V\left(q_{n}\right)-\lambda \\
& q_{n+1}=q_{n}
\end{aligned}
$$


and

$$
\begin{aligned}
p_{n+1} & =p_{n} \\
q_{n+1} & =q_{n}+\alpha p_{n+1} .
\end{aligned}
$$

We recall that, in our context, a volume preserving map is exact when $F^{*}\left(p d q_{1} \wedge d q_{2} \wedge \ldots \wedge d q_{d}\right)=p d q_{1} \wedge d q_{2} \wedge \ldots \wedge d q_{d}+d P$ where $P$ is $d-1$ form.

Indeed, (38) is an exact volume preserving map if and only if $\lambda=0$, since it is easy to observe that, when $\lambda=0$, both (40) and (41) are exact.

When $\varepsilon=0, \lambda=0$, the map (37) is integrable. The codimension-one tori given by $p=$ cte. are invariant and the motion in them is a rotation.

The volume preserving KAM theory leads us to expect that for $\epsilon \ll$ $1, \lambda=0$, the tori in which the frequency of the motion is Diophantine survive. We also expect that the tori with resonance, breaks down into lower dimensional tori. The lower dimensional tori can survive for $|\lambda| \ll 1$ (depending on $\epsilon$ ).

In this paper we have quantitative (but formal and non-rigorous) prediction of these phenomenon based on perturbative expansions. We hope that some of them may be either verified by rigorous results or explored numerically.

5.1. On the global geometry of the constraints given by (39). Integrable systems with constraints have been studied extensively in geometric mechanics. Nevertheless, the systems we consider here have some unusual properties that we would like to highlight.

It is customary to classify the constraints in holonomic when the distributions are integrable (in the sense that they foliate the phase space with a smooth quotient) and non-holonomic when the distributions are not integrable and they violate the hypothesis of Frobenius Theorem [Sou97, Aud08, Hol11].

The constraints (39) escape this dichotomy. They are locally integrable (they do satisfy the hypothesis of Frobenius Theorem and are locally given by invariant manifolds that give rise to a foliation) but nevertheless, the manifolds are dense, so that they do not give a nice quotient manifold.

Hence, even if we have holonomic constraints locally (and the infinitesimal results about holonomic systems are applicable), some global aspects such as symplectic reduction [Mey73, MW74, MW01] cannot be applied to (37).

5.2. Lyapunov exponents and phonon localization. In this section we study the so called phonon gap around the equilibria of (10) given by a hull function. 
Let us start by recalling some standard definitions. The main idea is that sound waves are defined by the propagation of infinitesimal disturbances around an equilibrium equation.

If we linearize around an equilibrium solution $x=\left\{x_{n}\right\}_{n \in \mathbb{Z}}$, we obtain the dynamics of the infinitesimal perturbations $\xi_{n}$ is given by

$$
\ddot{\xi}_{n}=\xi_{n+1}+\xi_{n-1}-2 \xi_{n}+\left(\partial_{\alpha}\right)^{2} V\left(\alpha x_{n}\right) \xi_{n} \equiv\left(\mathcal{L}_{x} \xi\right)_{n}
$$

It is clear that the propagation properties of sound waves will be affected by the spectral properties of the operator $\mathcal{L}_{x}$.

Note that the operator $\mathcal{L}$ is a one-dimensional Schrödinger operator with a position dependent potential. The dependence will be given by the dynamics of the $x_{n}$. In particular, for the solutions given by a hull function, we will be considering quasi-periodic potentials.

The mathematical theory of the spectrum of quasi-periodic Schrödinger operators is well developed [PF92, dlLH10]. In particular, it is known that the spectrum is independent of the $\ell^{p}$ space in which it is considered, and, more important for us, that the spectrum can be characterized by the existence of approximate eigenfunctions. In the dynamical interpretation in this section, the spectrum corresponds to the Lyapunov exponents of the solution [AMB92].

In the case of (37), we can study the Lyapunov spectra for any orbit using the geometric constraints (39).

Proposition 4. Let $\left(p_{n}, q_{n}\right)$ be an orbit of the mapping given by (37). Assume that it is an orbit in the full measure set that Osledets Theorem applies. Then, $d-1$ Lyapunov exponents are zero. Also, the sum of all the Lyapunov exponents is zero.

Proof. Consider $\tilde{F}$, the lift of the map $F$ in (38).

Let $s$ be a vector perpendicular to $\alpha$. It is a simple computation to show that:

$$
\tilde{F}\left(\tilde{M}_{q_{0}+s}\right)=\tilde{M}_{q_{0}}+s .
$$

Then it is clear that the $d-1$ vectors in the directions perpendicular to $s$ do not grow.

The fact that the sum of the Lyapunov exponents for orbits of a volume preserving map is zero is well known since the sums of the Lyapunov exponents is the rate of growth of the determinant of iterates of the map.

Of course, the dynamical system (37) is straightforward to implement numerically and allows study of statistical properties of depinning. 


\section{ACKNOWLEDGEMENTS}

We thank Dr. T. Blass for discussions. R. L. and L. Z. have been supported by DMS-1500943. The hospitality of JLU-GT Joint institute for Theoretical Sciences for the three authors was instrumental in finishing the work. R.L also acknowledges the hospitality of the Chinese Acad. of Sciences. X. Su is supported by both National Natural Science Foundation of China (Grant No. 11301513) and "the Fundamental Research Funds for the Central Universities".

\section{REFERENCES}

[AMB92] S. Aubry, R. S. MacKay, and C. Baesens. Equivalence of uniform hyperbolicity for symplectic twist maps and phonon gap for Frenkel-Kontorova models. Phys. D, 56(2-3):123-134, 1992.

[AP10] José Aliste-Prieto. Translation numbers for a class of maps on the dynamical systems arising from quasicrystals in the real line. Ergodic Theory Dynam. Systems, 30(2):565-594, 2010.

[Aud08] Michèle Audin. Hamiltonian systems and their integrability, volume 15 of SMF/AMS Texts and Monographs. American Mathematical Society, Providence, RI; Société Mathématique de France, Paris, 2008. Translated from the 2001 French original by Anna Pierrehumbert, Translation edited by Donald Babbitt.

$\left[\mathrm{BCH}^{+} 06\right]$ Martin Bücker, George Corliss, Paul Hovland, Uwe Naumann, and Boyana Norris, editors. Automatic differentiation: applications, theory, and implementations, volume 50 of Lecture Notes in Computational Science and Engineering. Springer-Verlag, Berlin, 2006. Papers from the 4th International Conference on Automatic Differentiation held in Chicago, IL, July 20-24, 2004.

[BdIL13] Timothy Blass and Rafael de la Llave. The analyticity breakdown for FrenkelKontorova models in quasi-periodic media: numerical explorations. J. Stat. Phys., 150(6):1183-1200, 2013.

[BK04] O. M. Braun and Y. S. Kivshar. The Frenkel-Kontorova model. Texts and Monographs in Physics. Springer-Verlag, Berlin, 2004. Concepts, methods, and applications.

[Car95] Henri Cartan. Elementary theory of analytic functions of one or several complex variables. Dover Publications Inc., New York, 1995. Translated from the French, Reprint of the 1973 edition.

[Chi79] Boris V. Chirikov. A universal instability of many-dimensional oscillator systems. Phys. Rep., 52(5):264-379, 1979.

[Die71] Jean Dieudonné. Infinitesimal calculus. Hermann, Paris, 1971. Translated from the French.

[dlL08] Rafael de la Llave. KAM theory for equilibrium states in 1-D statistical mechanics models. Ann. Henri Poincaré, 9(5):835-880, 2008.

[dlLH10] R. de la Llave and A Haro. Spectral theory and dynamics. 2010. Manuscript.

[dlLSZ15] Rafael de la Llave, Xifeng Su, and Lei Zhang. Resonant equilibrium configurations in quasi-periodic media: KAM theory. preprint, 2015.

[Fed75] Herbert Federer. Real flat chains, cochains and variational problems. Indiana Univ. Math. J., 24:351-407, 1974/75. 
[GGP06] Jean-Marc Gambaudo, Pierre Guiraud, and Samuel Petite. Minimal configurations for the Frenkel-Kontorova model on a quasicrystal. Comm. Math. Phys., 265(1):165-188, 2006.

[GPT13] Eduardo Garibaldi, Samuel Petite, and Philippe Thieullen. Discrete weak-kam methods for stationary uniquely ergodic setting. preprint, 2013.

[Har11] Alex Haro. Automatic differentiation methods in computational dynamical systems: invariant manifolds and normal forms. 2011.

[Hol11] Darryl D. Holm. Geometric mechanics. Part I. Imperial College Press, London, second edition, 2011. Dynamics and symmetry.

[KO13] Markus Kunze and Rafael Ortega. Twist mappings with non-periodic angles. 2065:265-300, 2013.

[LS03] Pierre-Louis Lions and Panagiotis E. Souganidis. Correctors for the homogenization of Hamilton-Jacobi equations in the stationary ergodic setting. Comm. Pure Appl. Math., 56(10):1501-1524, 2003.

[Mey73] Kenneth R. Meyer. Symmetries and integrals in mechanics. In Dynamical systems (Proc. Sympos., Univ. Bahia, Salvador, 1971), pages 259-272. Academic Press, New York, 1973.

[Mos67] Jürgen Moser. Convergent series expansions for quasi-periodic motions. Math. Ann., 169:136-176, 1967.

[MW74] Jerrold Marsden and Alan Weinstein. Reduction of symplectic manifolds with symmetry. Rep. Mathematical Phys., 5(1):121-130, 1974.

[MW01] Jerrold E. Marsden and Alan Weinstein. Comments on the history, theory, and applications of symplectic reduction. In Quantization of singular symplectic quotients, volume 198 of Progr. Math., pages 1-19. Birkhäuser, Basel, 2001.

[PF92] Leonid Pastur and Alexander Figotin. Spectra of random and almost-periodic operators, volume 297 of Grundlehren der Mathematischen Wissenschaften [Fundamental Principles of Mathematical Sciences]. Springer-Verlag, Berlin, 1992.

[SdlL12a] Xifeng Su and Rafael de la Llave. KAM theory for quasi-periodic equilibria in 1D quasi-periodic media: II. Long-range interactions. J. Phys. A, 45(45):455203, 24, 2012.

[SdlL12b] Xifeng Su and Rafael de la Llave. KAM Theory for Quasi-periodic Equilibria in One-Dimensional Quasi-periodic Media. SIAM J. Math. Anal., 44(6):39013927, 2012.

[Se192] W Selke. Spatially modulated structures in systems with competing interactions. In Phase transitions and critical phenomena, Volume 15, pages 1-72. Academic Press, 1992.

[Sou97] J.-M. Souriau. Structure of dynamical systems, volume 149 of Progress in Mathematics. Birkhäuser Boston, Inc., Boston, MA, 1997. A symplectic view of physics, Translated from the French by C. H. Cushman-de Vries, Translation edited and with a preface by R. H. Cushman and G. M. Tuynman.

[vEF02] T. S. van Erp and A. Fasolino. Aubry transition studied by direct evaluation of the modulation functions of infinite incommensurate systems. Europhys. Lett., 59(3):330-336, 2002.

[vEFJ01] T. S. van Erp, A. Fasolino, and T. Janssen. Structural transitions and phonon localization in Frenkel Kontorova models with quasi-periodic potentials. Ferroelectrics, 250:421-424, 2001. 
[vEFRJ99] T. S. van Erp, A. Fasolino, O. Radulescu, and T. Janssen. Pinning and phonon localization in Frenkel-Kontorova models on quasiperiodic substrates. Physical Review B, 60(9):6522-6528, 1999.

School of Mathematics, Georgia Institute of Technology, 686 Cherry St., Atlanta GA 30332, USA

JLU-GT joint institute for Theoretical Science, Jilin University, Changchun, 130012, CHINA

E-mail address: rafael.delallave@math.gatech.edu

School of Mathematical Sciences, Beijing Normal University, No. 19, XinJieKouWai St.,HaiDian District, Beising 100875, P. R. China

E-mail address: xfsu@bnu.edu.cn

School of Mathematics, Georgia Institute of Technology, 686 Cherry St., Atlanta GA 30332, USA

E-mail address: 1zhang98@math.gatech.edu 